Landslides (2016) 13:1319

DOI 10.1007/s10346-015-0653-7

Published online: 20 November 2015

○) Springer-Verlag Berlin Heidelberg 2015

Silvia Salas-Romero · Alireza Malehmir · Ian Snowball · Bryan C. Lougheed · Magnus Hellqvist

\title{
Erratum to: Identifying landslide preconditions in Swedish quick clays-insights from integration of surface geophysical, core sample- and downhole property measurements
}

Erratum to: Landslides

DOI 10.1007/s10346-015-0633-y

The original version of this article, unfortunately, contained errors. Listed below are the necessary changes to the paper.

1. In the "Methodology" section, under "Field data acquisition and sampling" subsection

The texts:

(about $1 \mathrm{~cm}$ thickwall thickness)

Should be:

(about $1 \mathrm{~cm}$ wall thickness)

2. In the "Methodology" section, under "Laboratory measurements" subsection, and under item "6 X-ray powder diffraction"

The texts:

incident angle between $3^{\circ}$ and $70^{\circ} 20$

Should be:

incident angle between $3^{\circ}$ and $70^{\circ} 2 \theta$
The texts:

$35^{\circ} 20$, with a step size of $0.02^{\circ} 20 / \mathrm{s}$

Should be:

$35^{\circ} 2 \theta$, with a step size of $0.02^{\circ} 2 \theta / \mathrm{s}$

The online version of the original article can be found at http://dx.doi.org/10.1007/ s10346-015-0633-y.

S. Salas-Romero $(-2) \cdot$ A. Malehmir $\cdot$ I. Snowball · B. C. Lougheed $\cdot$ M. Hellqvist Department of Earth Sciences,

Uppsala University,

Villavägen 16, 75236, Uppsala, Sweden

e-mail: silvia.salas_romero@geo.uu.se

B. C. Lougheed

Department of Geology,

Lund University,

Sölvegatan 12, 22362, Lund, Sweden 